Presented by G.Wilk at Strangeness'96 Workshop, Budapest 1996, to be published in Heavy Ion Phys. (1996)
S'96

Budapest

(c) May 15-17, 1996

\title{
Propagation of Strange Quark Matter in the Atmosphere
}

\author{
Grzegorz Wilk ${ }^{1}$ and Zbigniew Włodarczyk ${ }^{2}$ \\ 1 Soltan Institute for Nuclear Studies, \\ 00-681 Warsaw, Poland \\ 2 Institute of Physics, Pedagogical University, \\ 25-509 Kielce, Poland
}

As for 30 June 1996.

\begin{abstract}
We propose model of propagation of lumps of Strange Quark Matter (strangelets) through the atmosphere, which accounts for their apparent strong penetrability and normal nuclear-type sizes at the same time. The mass spectrum of strangelets reaching the Earth predicted by this model agrees very well with the existing data on the abundance of different elemets in the Universe.
\end{abstract}

\section{Introduction}

In astrophysical literature one finds a number of phenomena which can be regarded as possible manifestation of the strange quark matter (SQM), extremaly interesting possibility of new stable form of matter [1, 2, 3, 4. 4. There were attempts to find lumps of SQM called strangelets in the terrestial experiments devoted to search for Quark Gluon Plasma but so far without success [7] (what can be interpreted that no QGP was formed so far in these experiments for also in cosmic ray (CR) events [9, 10, 11, 12, 13, the most famous candidate being the so called Centauro events 14. In CR one either deals with strangelets formed by some astrophysical mechanisms [1], 15] or one witnesses their production proceeding in collisions of the original CR flux with atmospheric nuclei [16].

Centauro events are good example of problem being the topic of our presentation. They were observed very deep in the atmosphere (at $\sim 500 \mathrm{~g} / \mathrm{cm}^{2}$ ) and if they would be really caused by strangelets [16], it would require unusual penetrability of such objects implying their small geometrical sizes (much smaller than the typical nuclear size). Similar conclusions were also reached in some CR experiments [12, 13. We present an alternative approach to the propagation of strangelets through the atmosphere in which we argue that they have most probably normal nuclear-type sizes and similar propagation properties. We then demonstrate how such strangelets can penetrate deeply in the atmosphere producing effects ascribed before to higly penetrating objects. Finally, we estimate the initial flux of strangelets entering atmosphere emerging from our picture and compare it to the known abundances of normal nuclei in Universe. 


\section{Strangelets}

Typical SQM consists of roughly equal number of $u, d$ and $s$ quarks and it has been found to be the true ground state of QCD [1, 2, 3], i.e., it is absolutely stable at high mass numbers $A$. However, any SQM produced at very early stage of the history of Universe would have evaporated long time ago 4 . On the other hand, there are places where SQM may still exist at present [1, 1. It is probably continously produced in neutron stars with a superdense quark surface and in quarks stars with a thin nucleon envelope 115, 11. Collisions of such objects could therefore produce small lumps of SQM, strangelets with $10^{2}<A<10^{6}$, permeating the Galaxy and possibly reaching also the Earth (i.e., a priori being detectable here).

The practical measure of the stability of strangelet is provided by the so called separation energy $d E / d A$, i.e., energy, which is required to remove a single baryon from a strangelet. For example, if $d E / d A>m_{N}$ then strangelet can evaporate neutrons from its surface. This energy depends, among other things, on the size of strangelet, i.e., on its mass number $A$ [3]. There exists therefore some critical size $A=A_{\text {crit }}(\sim 300-400$ depending on the parameters used $)$ such that for $A<A_{\text {crit }}$ strangelets are unstable against neutron emission [3].

Anomalous massive particles, which can be interpreted as strangelets, have been apparently observed in three independent CR experiments:

(i) In counter experiment devoted to study primary CR nuclei two anomalous events have been observed [9]. They are consistent with values of charge $Z \simeq 14$ and of mass numbers $A \simeq 350$ and $\simeq 450$ and cannot be accounted for by the conventional background. Such values of $Z$ and $A$ are fully consistent with the theoretical estimation for ratio $Z / A$ in SQM 17 .

(ii) The so called Price's event [10] with $Z \simeq 46$ and $A>1000$, regarded previously as possible candidate for magnetic monopole, turned out to be fully consistent with the above ratio $Z / A$ for SQM [18].

(iii) The so called Exotic Track with $Z \simeq 20$ and $A \simeq 460$ has been reported in 11. The name comes from the fact that although it was observed in emulsion chamber exposed to $\mathrm{CR}$ on balloon at the atmospheric depth of only $11.7 \mathrm{~g} / \mathrm{cm}^{2}$ its arrival zenith angle of $87.4 \mathrm{deg}$ means that the projectile causing that event traversed $\sim 200 \mathrm{~g} / \mathrm{cm}^{2}$ of atmosphere (in contract to events $(i)$ and (ii) where the corresponding depths were of the order of $5-15 \mathrm{~g} / \mathrm{cm}^{2}$ of atmosphere only).

The Exotic Track event motivated the (balloon born emulsion chamber) JACEE 12] and Concorde aircraft [13] experiments to search for strangelets with such long mean free paths. In fact, authors of 12, 13] suggest that the interaction mean free path for strangelets in atmosphere is of the order of $\lambda_{\mathrm{S}}=124 \mathrm{~g} / \mathrm{cm}^{2}$ for $A=100$ decreasing to $\lambda_{\mathrm{S}}=59 \mathrm{~g} / \mathrm{cm}^{2}$ only for $A=1000$. These values are surprisingly close to that for protons at comparable energies $\left(\lambda_{\text {proton }}=60-70 \mathrm{~g} / \mathrm{cm}^{2}\right)$ and are much bigger than that for a normal nucleus $\left(\lambda_{\text {nucleus }} \simeq 3.8 \mathrm{~g} / \mathrm{cm}^{2}\right.$ for $\left.A=100\right)$ [19]. It was then suggested in 12, 13 that strangelets should have geometrical radii much smaller than those of the ordinary nuclei or, correspondingly, much smaller inter- 
action cross sections (in agreement with SQM interpretation of Centauro events mentioned before [16]).
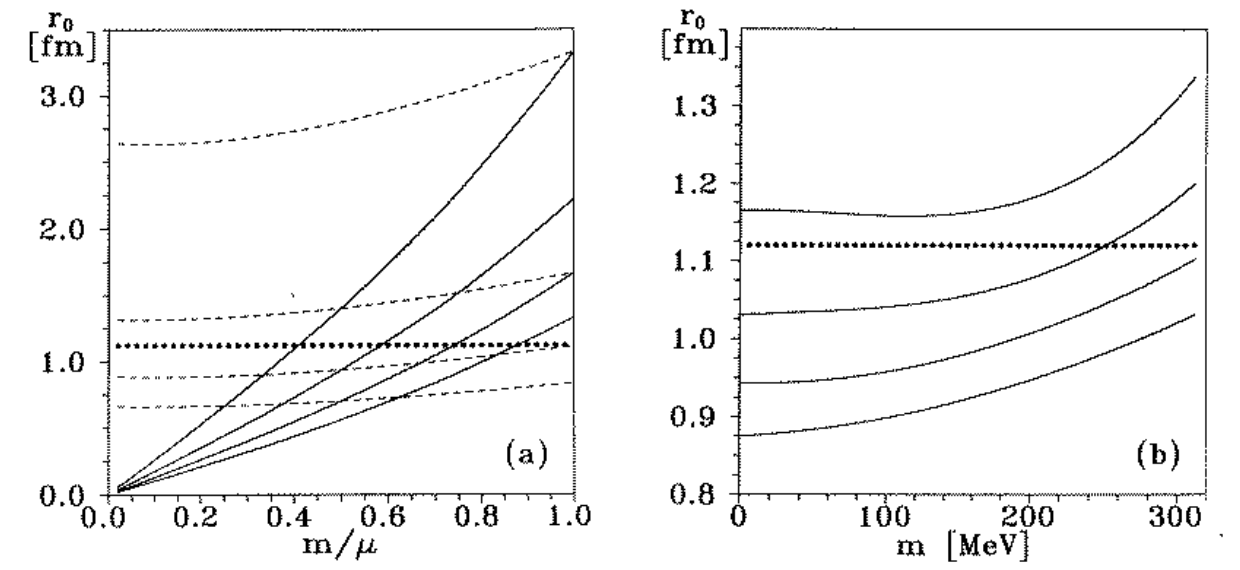

Fig. 1. Dependence of the rescaled radius $r_{0}$ [fm] (eq.(1D)): (a) on the ratio of $s$ quark mass $m$ to its chemical potential $\mu$, $\mathrm{m} / \mu$ (solid lines, read from top to bottom, correspond to fixed $m=100,150,200,250 \mathrm{MeV}$; dashed read from top to bottom correspond to fixed $\mu=100,200,300,400 \mathrm{MeV}$ ); (b) on the strange quark mass $m$ for different values of $\alpha_{c}=0.9,0.6,0.3,0.0$ (from top to bottom, respectively). In both cases dotted line shows $r_{0}=1.12 \mathrm{fm}$ corresponding to normal nuclear density $\rho=0.17 \mathrm{fm}^{-3}=(110 \mathrm{MeV})^{3}$.

However, such expectations are not confirmed by calculations, cf. Fig. 1. Let us consider a strangelet visualised as Fermi gas of $u, d$ and $s$ quarks, with total mass number $A$, confined in spherical volume $V \sim A$ of radius $R=r_{0} A^{1 / 3}$, where the rescaled radius $r_{0}$ is determined by the number density of strange matter, $n=A / V=\left(n_{u}+n_{d}+n_{s}\right) / 3$, for which $r_{0}=(3 / 4 \pi n)^{1 / 3}$. Here $n_{i}=-\frac{\partial \Omega_{i}}{\partial \mu_{i}}$ and thermodynamical potentials $\Omega_{i}\left(m_{i}, \mu_{i}\right)$ are related to chemical potentials $\mu_{i}$ [3]. Because in our case all $\mu_{i} \sim 300 \mathrm{MeV}$, one can neglect the (current) masses of $u$ and $d$ quarks and keep only the $s$ quark mass $m$. Taking into account the QCD $\mathcal{O}\left(\alpha_{c}\right)$ corrections in $\Omega_{i}\left(m_{i}, \mu_{i}, \alpha_{c}\right)$ and renormalizing them at $m_{N} / 3=313 \mathrm{MeV}$ [3], one arrives at

$$
r_{0}=\left\{\frac{3 \pi}{2\left(1-\frac{2 \alpha_{c}}{\pi}\right)\left[\mu^{3}+\left(\mu^{2}-m^{2}\right)^{3 / 2}\right]}\right\}^{1 / 3} .
$$

As one can see in Fig. $1 a$ for $m \simeq 150 \mathrm{MeV}$ and $\mu \simeq 300 \mathrm{MeV}$ (the values commonly accepted for SQM [1, 3]), the values of $r_{0}$ of the strangelets are comparable with 
those for the ordinary nuclear matter. Fig. $1 b$ summarizes dependence of $r_{0}$ on $\alpha_{c}$. As one can see QCD corrections lead to slight increase of $r_{0}$. (It is worth to say that at this point we differ drastically from previous works looking at strangelets from the QGP and Centauro production perspective [16] where large value of $\mu$ was the main reason for small values of $r_{0}$ and for all further claims concerning large penetrability of strangelets. Such large values of $\mu$ were justified there because strangelets were supposed to be produced from the QGP phase formed in baryon rich environment whereas in our case they are supposed to be stable object entering Earth atmosphere as a part of $\mathrm{CR}$ radiation.). Fig. 1 shows therefore that the expected decrease of the radius of strangelet is nowhere as dramatic as it has been estimated in Refs. [12, 13], i.e., the expected geometrical cross sections of SQM are not able to explain alone the occurences of anomalous events detected deeply in the atmosphere.

\section{Possible scenario of propagation of strangelets through the atmosphere}

It does not mean, however, that some form of SQM does not reach Earth and penetrates deep in the atmosphere to be finally registered. The apparent contradiction between its "normal" size and strong penetrability can be resolved in very simple manner. In what follows we shall propose the respective (speculative but plausible) scenario, which can be summarized in the following way: Strangelets reaching so deeply into atmosphere are formed in many successive interactions with air nuclei of much heavier lumps of SQM entering our atmosphere. In this way one can accomodate both the most probably "normal" mean free paths for successive interactions and final large penetrating depth. Such scenario is fully consistent with all present and proposed experiments and could be additionally checked only by measuring products of the intermediate collisions, which so far is impossible.

To provide numerical estimations we shall limit ourselves to two extreme pictures of collisions of strangelet of mass number $A$ with air nucleus target of mass number $A_{t}$ :

1. "Standard model": all quarks of $A_{t}$ which are located in the geometrical intersection of two colliding projectiles are involved and it is assumed that each quark from the target interacts with only one quark from the strangelet; i.e., during interaction $3 A_{t}$ quarks from strangelet can be used up and after it the mass number of strangelet is diminished to the value of $A-A_{t}$ at most. In this case simple analytical estimation is possible leading to the total penetration depth of strangelet

$$
\Lambda \simeq \frac{1}{3} \lambda_{N A_{t}}\left(\frac{A_{0}}{A_{t}}\right)^{1 / 3}\left(1-\frac{A_{\text {crit }}}{A_{0}}\right)^{2}\left(4-\frac{A_{\text {crit }}}{A_{0}}\right) \cong \frac{4}{3} \lambda_{N A_{t}}\left(\frac{A_{0}}{A_{t}}\right)^{1 / 3} .
$$

(where $\lambda_{N A_{t}}$ denotes the mean free path for $N A_{t}$ collisions).

2. "Tube-like model": All quarks from both nuclei which are in their geometrical 
intersection region participate in the collision. This is an analogue of the so called tube model (TM) used sometimes in nuclear collisions and after collision the atomic mass of strangelet diminishes to the value equal to $A-A^{1 / 3} \cdot A_{t}^{2 / 3}$ (this variant should be regarded as a limiting possibility providing estimation of the maximal possible destruction of the quarks in the strangelet).
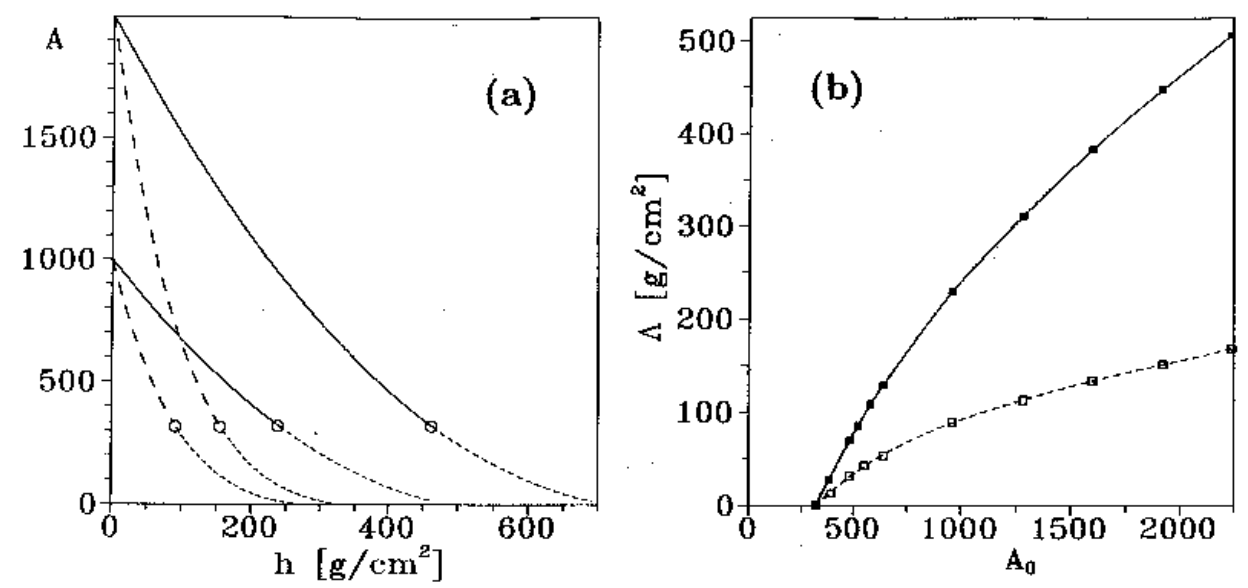

Fig. 2. (a) An example of the predicted decrease of actual size of strangelet $A$ with depth $h$ of atmosphere traversed for two different initial sizes: $A_{0}=1000$ and 2000 and for two scenarios of interaction: standard (solid and dotted lines) and tube-like (dashed and dotted lines). In both scenarios dotted lines correspond to $A<A_{\text {crit }}$. (b) Atmospheric length $\Lambda$ after which initial strangelet reaches its critical dimension, $A=A_{\text {crit }}(=320)$ drawn as a function of its initial mass number $A_{0}$ for standard (solid line) and tube-like (dashed line) interaction. Consecutive squares indicate points where (for $\left.A_{0}>600\right) A_{0} / A_{\text {crit }}=k,(k=2,3, \ldots)$.

In the numerical calculations we take $A_{t}=14.5$ for the atmosphere (assuming $25 \%$ of oxygen and $75 \%$ of nitrogen) and neglect the influence of the interstellar gas. As a result one obtains the mass number of strangelet registered at depth $h, A(h)$, as a function of $h$ as shown in Fig. $2 a$. As one can see, bigger initial strangelets (i.e., with higher mass number $A_{0}$ ) can penetrate much more deeply into atmosphere untill $A(h)$ exceeds critical $A_{\text {crit }}$, after which point they just evaporate by the emission of neutrons. Notice much stronger degradation of the initial strangelets for the case of the tube-like model interactions with air nuclei. As we have already mentioned, our scheme displays two most extreme situations in order to provide limits for all further possible estimations. Because, for simplicity, we tacidly assume that air nucleus destroys totally only the corresponding (equal to it) part of the 
incoming strangelet, our estimation provides therefore only a lower limit of what should be expected in more detailed calculations. More refined estimations should take into account also the surface tension effects and the possible dependence of $r_{0}$ and cross section on the actual mass number of strangelet $A(h)$, which translates immediately into dependence on $h$. However, their effect would be of secondary importance only and at present experimental situation such detailed and tedious estimations would be simply premature.
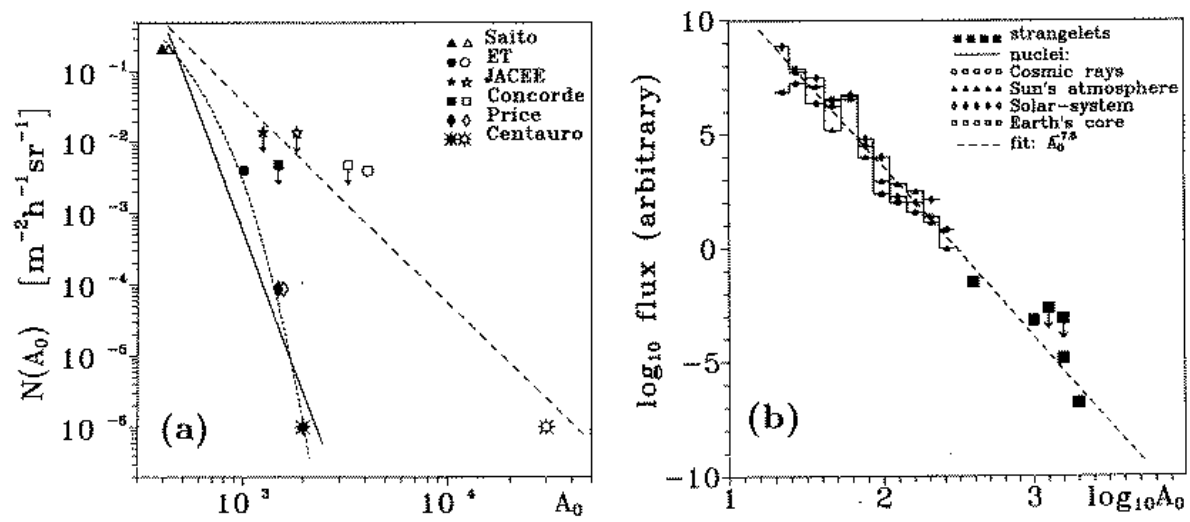

Fig. 3. (a) The estimation of the expected flux of strangelets on the border of atmosphere, $N\left(A_{0}\right)$, as a function of their mass number as obtained from standard (full symbols; solid line $\sim A_{0}^{-7.5}$ and dotted line $\sim \exp \left(-A_{0} / 130\right)$ ) and tube-like (empty symbols; dashed line $\sim A_{0}^{-2.8}$ ) models. The respective points are taken from: Saito [9, ET [18], Centauro [14], JACEE [12], Concorde 13 and Price 10]. See text for further details. (b) Comparison of $N\left(A_{0}\right)$ from $(a)$ with the abundance of elements in the Universe after [21].

Suppose now that the energy per baryon in strange matter is $\varepsilon=919 \mathrm{MeV}$ and that number densities corresponding to nuclear matter are $n=(110 \mathrm{MeV})^{3}$ [3]. In such situation, for $A \leq 1100, E / A$ exceeds already $m_{N}$ but strangelet does not emit neutrons yet and starts to do so only for $A \leq 320$, at which point $d E / d A$ exceeds $m_{N}$ [3]. Below this limit strangelet decays rapidly by evaporating neutrons. In view of these remarks it is remarkable that all possible candidates for SQM have mass numbers near or slightly exceeding $A_{\text {crit }}$, namely: $A=350$ and 450 in [9], $A=460$ in [11] and $A=1000$ in [18] and it is argued that Centauro event contains probably $\sim 200$ baryons $[14$.

Fig. $2 b$ shows atmospheric length traversed after which strangelet mass number $A$ becomes critical, $A=A_{\text {crit }}$ (starting from different mass numbers $A_{0}$ of the 
initial strangelets). From this figure one can read off that strangelets which are observed at depth $200 \mathrm{~g} / \mathrm{cm}^{2}$ should originate from strangelets of mass number $A_{0}=900$ at the top of the atmosphere whereas Centauro events observed at the mountain altitudes would require original strangelet of $A_{0}=1800$.

Let us now estimate flux of strangelets reaching our atmosphere starting with the experimental data taken at different atmospheric depths, cf. Fig. 3a. The experimental data Saito, ET and Centauro on measured fluxes on different atmospheric depths (which can be interpreted in terms od SQM) are taken from [9], [18] and [14], respectively. The corresponding upper limits (no strangelets found so far), JACEE and Concorde, are from 12. and 13. Notice that Price data 10 (assuming that what was observed was indeed a strangelet) favour standard model. In terms of fits (for 3 points: Saito, ET, Centauro) one gets: $\sim A_{0}^{-7.5}$ for standard model (full line, dotted line corresponds to $\sim \exp \left(-A_{0} / 130\right)$ in this model) and $\sim A_{0}^{-2.8}$ for tube-like model (dashed line). The choice of the power-like or exponential form for standard model was dictated by the analogy to nuclear fragmentation and the expectation that decay (fragmentation) of a strange star after its collision will result in the production of strangelets with similar distribution of mass 20. It is interesting to note that essentially the same exponent, $A_{0}^{-7.5}$, as obtained in the standard model fit is observed also for occurence of normal nuclei in the Universe [21], cf. Fig. 3b.

\section{Summary and conclusions}

We summarize by stating that most probable the geometrical cross sections of strangelets are not dramatically different from those for the ordinary nuclear matter and cannot therefore explain their apparent very high penetrability through the atmosphere. Instead we propose to interprete such a penetrability of strangelets (already discovered or to be yet observed) as indication of the existence of very heavy lumps of SQM entering our atmosphere, which are then decreased in size during their consecutive collisions with air nuclei (i.e., their original mass number $A_{0}$ is reduced until $A=A_{\text {crit }}$ ) and finally decay be the evaporation of neutrons. Assuming that mass distribution of strangelets comprising this initial flux follows power low $A_{0}^{\gamma}$ we estimate that $\gamma \geq 2$ is consistent with present experimental data. Our result is consistent with other estimations of the flux of strangelets at different atmospheric depths. In particular it is interesting to note that the best fit to data representing this dependence comes from our standard model and it agrees very well with the existing data on chemical composition of normal nuclei supporting therefore our hypothesis that geometrical sizes of strangelets are not much different from those of normal nuclei of the same $A$.

\section{Acknowledgement}

One of the Authors (G.W) is deeply gratefull to Profs. T. Csörgö, P.Levai and J. 
Zimanyi for making his participation in Strangeness'96 conference possible and for its warm and extremaly fruitful atmosphere.

\section{References}

1. E.Witten, Phys. Rev. D30 (1984) 272. Cf. also: Proc. of the Int. Workshop on SQM in Physics and Astrophysics, eds. J.Madsen and P.Haensel, Nucl. Phys. B24 (Proc. Suppl.) (1991) and Proc. of the Int. Symp. on Strangeness And Quark MatTer, Kolymbari, Greece, Sept. 1-5, 1994, World Scientific Pub. 1995.

2. C.Alcock and A.Olinto, Ann. Rev. Nucl. and Part. Phys. 38 (1988) 161.

3. E.Farhi and R.L.Jaffe, Phys. Rev. D30 (1984) 2379; M.S.Berger and R.L.Jaffe, Phys. Rev. C35 (1987) 213.

4. C.Alcock and E.Farhi, Phys. Rev. D32 (1985) 1273; E.Farhi, Comm. Nucl. Part. Phys. 16 (1986) 289.

5. G.Baym et al., Phys. Lett. B160 (1985) 181; G.Shaw, G.Benford and D.Silverman, Phys. Lett. 169 (1986) 275.

6. C.Alcock, E.Farhi and A.V.Olinto, Phys. Rev. Lett. 57 (1986) 2088.

7. K.Borer et al., Phys. Rev. Lett. 72 (1994) 1415; D.Bevais et al. Phys. Rev. Lett. 75 (1995) 3078. Also more dedicated experiment by J.Vandegriff et al., Phys. Lett. B365 (1996) 418, failed to observe strangelets in terrestial atmosphere. For older propositions and results see [1].

8. C.Spieles et al., Phys. Rev. Lett. 76 (1996) 1776 and references therein.

9. T.Saito, Y.Hatano and Y.Fukada, Phys. Rev. Lett. 65 (1990) 2094.

10. P.B.Price et al., Phys. Rev. D18 (1978) 1382.

11. M.Ichimura et al., Nuovo Cim. A106 (1993) 843.

12. O.Miyamura et al., Proc. $24^{\text {th }}$ ICRC 1, Rome (1995) 890.

13. J.N.Capdevielle et al., Proc. $24^{\text {th }}$ ICRC 1, Rome (1995) 910.

14. C.M.G.Lattes, Phys. Rep. 65 (1980) 151.

15. P.Haensel et al., Astron. Astrophys. 160 (1986) 121 and Astrophys. Journal 310 (1986) 261.

16. A.D.Panagiotou A.Petridis and M.Vassiliou, Phys. Rev. D45 (1992) 3134; M.N.Asprouli, A.D.Panagiotou and E.Gładysz-Dziaduś, Astropart. Phys. 2 (1994) 167.

17. M.Kasuya et al., Phys. Rev. D47 (1993) 2153.

18. T.Saito, Proc. $24^{\text {th }}$ ICRC 1, Rome (1995) 898.

19. So far the results are negative: no evidence for strangelets with $Z>26$ that survived passing of $\sim 100 \mathrm{~g} / \mathrm{cm}^{2}$ of atmosphere was found.

20. Cf., for example, percolation model of J.Nemeth et al., Z.Phys. A325 (1986) 347 and references therein.

21. G.B.Zhdanov, Usp. Fiz. Nauk 111 (1973) 109 [Sov. Phys. Uspekhi 16 (1974) $642]$. 